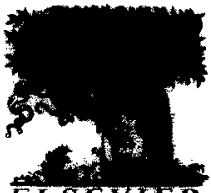

Early Human Development 41 (1995) 111-127

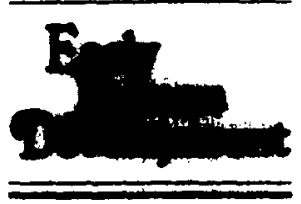

\title{
Perinatal exposure to polychlorinated biphenyls and dioxins and its effect on neonatal neurological development
}

\author{
Marcel Huisman*a, Corine Koopman-Esseboom ${ }^{\mathrm{b}}$, Václav Fidler ${ }^{\mathrm{c}}$, \\ Mijna Hadders-Algra ${ }^{\mathrm{d}}$, Cornelis G. van der Paauw ${ }^{\mathrm{e}}$, \\ Louis G.M.Th. Tuinstra ${ }^{f}$, Nynke Weisglas-Kuperus ${ }^{\mathrm{b}}$, \\ Pieter J.J. Sauer ${ }^{b}$, Bert C.L. Touwen ${ }^{g}$, E. Rudy Boersma ${ }^{a}$ \\ Department of Obstetrics and Gynaecalogy, Nutrition and Development Unit. \\ University Hospital, P.O. Box 30.001. NL-9700 RB Groningen, The Netherlands \\ 'Department of Pediatrics, Erasmus University and University Hospital/Sophia \\ Children's Hospital, Rotterdam. The Netherlands \\ 'Department of Health Sciences, Epidemiology and Statistics Unit, \\ University of Groningen, Groningen. The Netherlands \\ 'Department of Medical Physiology, Developmental Neurosciences, \\ University Hospital, Groningen, The Netherlands \\ 'TNO Nutrition and Food Research Institute, Zeist, The Netherlands \\ ${ }^{f}$ DLO State Institute for Quality Control of Agriculture Products, \\ Wageningen, The Netherlands \\ sDepartment of Medical Physiology, Developmental Neurology, \\ University Hospital, Groningen. The Netherlands
}

Received 30 September 1994; revision received 30 November 1994; accepled 5 December 1994

\begin{abstract}
Polychlorinated biphenyls (PCBs) and dioxins (polychlorinated dibenzo-p-dioxins (PCDDs), and dibenzofurans (PCDFs)) are widespread environmental contaminants which are neurotoxic in animals. Perinatal exposure to PCBs, PCDDs, and PCDFs occurs prenatally via the placenta and postnatally via breast milk. To investigate whether such an exposure affects the neonatal neurological condition, the neurological optimality of 418 Dutch newborns was evaluated with the Prechtl neurological examination. Half of the infants were breast-fed, the other half were formula-fed, representing a relatively high against a relatively low
\end{abstract}

\footnotetext{
* Corresponding author.
} 
postnatally exposed group, respectively. As an index of prenatal exposure, four non-planar PCBs in cord and maternal plasma were used. These PCB levels were not related to neurological function. As measures of combined pre- and early neonatal exposure, 17 dioxin congeners, three planar, and 23 non-planar PCB congeners were determined in human milk in the second week after delivery. Higher levels of PCBs, PCDDs, and PCDFs in breast milk were related to reduced neonatal neurological optimality. Higher levels of planar PCBs in breast milk were associated with a higher incidence of hypotonia. This study confirms previous reports about the neurotoxic effects of these compounds on the developing brain of newborn infants.

Keywords: Polychlorinated biphenyls; Dioxins; Optimality concept; Infant nutrition; Neonatal neurological condition

\section{Introduction}

Polychlorinated biphenyls (PCBs) and dioxins (polychlorinated dibenzo-p-dioxins (PCDDs), and dibenzofurans (PCDFs)) are polycyclic aromatic compounds that are widespread environmental contaminants [1]. They are toxic for animals and human beings. PCBs have a total number of 209 possible congeners that differ in degree of chlorination and chlorine position. PCBs have electrical insulating properties. They are resistant to high temperatures and can easily conduct heat. Consequently, they have been widely used as plasticizers, fire retardants, in carbonless copy paper, in hydraulic fluids and as dielectric fluids in capacitors and transformers. In the late 1970 s, they were banned world-wide because of their environmental persistence. However, production continued until the mid 1980s in e.g. the Czech Republic [2]. Dioxins are a mixture of possibly 75 PCDD and 135 PCDF congeners. They are unwanted byproducts of industrial and thermal processes. In the Netherlands, the incineration of municipal and industrial waste is the most important source for atmospheric deposition [3]. Since PCBs, PCDDs, and PCDFs are highly lipophilic and chemically stable [4], they tend to accumulate in the food chain. Considerable concentrations have been found in dairy products, fish, meat, and breast milk [5]. Food is the major source ( $>90 \%$ ) of human exposure to PCBs, PCDDs, and PCDFs [6].

Numerous animal studies have indicated that PCBs, PCDDs, and PCDFs are toxic to the nervous system [7-11]. Perinatal exposure of rats has been shown to induce effects on brain dopamine concentrations [12] and alterations in steroidal and thyroid hormone status [13].

In Japan in 1968 (Yusho incident) as well as in Taiwan in 1979 (Yu-cheng incident), about 2000 people were accidentally exposed to PCBs, PCDFs, and polychlorinated quaterphenyls through the consumption of contaminated rice oil. The most evident clinical sequelae were dermal lesions such as chloracne and comedones. PCBs and dioxins cross the placenta and can affect the developing fetus. The offspring of exposed women were small-for-gestational age and showed hyperpigmenta- 
tion of the skin and nails. In follow-up studies, these children demonstrated signs of apathy, hypotonia, and a low performance on intelligence tests $[14,15]$.

Two studies in the USA indicated toxic effects of prenatal exposure to background PCB levels. Jacobson et al. [16] followed 242 infants whose mothers consumed PCBcontaminated fish from Lake Michigan and 71 control infants whose mothers did not eat fish. In a study of 912 infants, Rogan et al. [17] evaluated the neonatal effects of transplacental exposure to PCBs. Both studies found a significant negative effect on neonatal behavioural performance, assessed with the Brazelton Neonatal Behavioral Assessment Scale (BNBAS) [18]. Until now effects have been evaluated behaviourally, and not with an age-adequate neurological examination. The present paper aims to fill this gap.

As in other industrialized countries in Western Europe, contamination of Dutch breast milk with PCBs, PCDDs, and PCDFs is high [19]. In 1989, this contamination of breast milk with high levels of possibly neurotoxic substances developed into a great public concern. As a result, the Dutch government initiated a prospective longitudinal study on possible adverse effects, starting in the neonatal period. In order to examine health risks associated with postnatal PCB and dioxin exposure, half of the study population was selected on the basis of the realized maternal intention to breast-feed their infants; the other half intended to formula-feed their infants. In formula-feeding, milk lipids are replaced by lipids of vegetable origin with a negligible content of PCBs and dioxins. An evaluation of the postnatal effects of PCB and dioxin exposure includes necessarily eventual effects caused by transplacental exposure during the prenatal period. PCB levels in cord and maternal plasma were used as a direct index of prenatal PCB exposure. PCB and dioxin levels in human milk are closely correlated with levels in maternal adipose tissue [5]. Therefore, PCB and dioxin levels in breast milk are good indicators of maternal load and subsequently of prenatal and postnatal exposure. In the present study, we report on the relationship between perinatal background exposure to PCBs, PCDDs, and PCDFs and the neurological condition of 418 Dutch newborns.

\section{Subjects and methods}

\subsection{Study design}

The study was carried out in two widely separated and different areas in the Netherlands: Groningen, a semi-urban area in the northeast, and Rotterdam, a highly industrialized region in the southwest of the Netherlands. Approval was given by the ethics committees of the Groningen and the Rotterdam university hospitals. Intake took place from June 1990 until June 1992. Two-hundred healthy pregnant women were planned to be included in each area: $50 \%$ who intended to breast-feed their infants for at least 6 weeks and $50 \%$ who preferred formula-feeding. The latter agreed to use Almiron M2 (Nutricia N.V., The Netherlands) from one batch as a reference. The women were asked to cooperate by their midwife or obstetrician and were visited at home for an explanation of the study protocol. The women and their children had to meet the following criteria: absence of serious illness and complications during pregnancy and delivery; caucasian race; first or second born term in- 
fants (37-42 weeks of gestation); no caesarian section; no forceps or vacuum extraction; availability of a maternal blood sample in the last month of gestation and of a cord blood sample.

\subsection{Obstetrical and neurological optimality scores}

Obstetrical data were evaluated according to the obstetrical optimality list, as described by Touwen et al. [20]. The list used in this study consisted of 72 items that measure socioeconomic situation and pre-, intra-, and immediate post-partum conditions. By counting the number of items that fulfilled preset criteria for optimality [21], the optimality score was calculated.

The neonatal neurological examination was scheduled between the 10th and the 21 st day after delivery. For the assessment of the neonatal neurological condition, the comprehensive age-adequate neurological examination, as described by Prechtl [22], was used. This technique, in contrast to the BNBAS [23], has proven to be predictive for later major and minor neurological dysfunctions [24-27]. The examination leads to a clinical diagnostic classification: normal, suspect, or abnormal. Furthermore, two clusters of items were formed: one describing postural tone, the other reflexes and responses. The latter cluster consisted of 11 items, the former consisted of 10 items (Table 1). For each item score, 0 represents a low value, score 1 an intermediate value and score 2 a high value as may occur in healthy vigorous infants. By summation of scores, a postural tone and a reflex cluster score were calculated which could range from $0-17$ and $0-22$, respectively. A postural tone

Table 1

The reflex and postural tone cluster score

\begin{tabular}{|c|c|c|c|c|c|c|c|}
\hline \multirow[t]{2}{*}{ Reflex cluster } & \multicolumn{3}{|c|}{ Score $^{a}$} & \multirow[t]{2}{*}{ Postural tone cluster } & \multicolumn{3}{|c|}{ Score $^{a}$} \\
\hline & 0 & 1 & 2 & & 0 & 1 & 2 \\
\hline Lip reflex & + & ++ & +++ & Overshooting movements & + & - & \\
\hline Glabella reflex & + & ++ & +++ & $\begin{array}{l}\text { Posture of arms in supine } \\
\text { suspension (extended) }\end{array}$ & + & - & \\
\hline Abdominal skin reflex & + & ++ & +++ & $\begin{array}{l}\text { Posture of legs in supine } \\
\text { suspension (extended) }\end{array}$ & + & - & \\
\hline Biceps reflex & + & ++ & +++ & $\begin{array}{l}\text { Resistance against passive } \\
\text { movements }\end{array}$ & + & ++ & +++ \\
\hline Knee reflex & + & ++ & +++ & Recoil of arms & + & ++ & +++ \\
\hline $\begin{array}{l}\text { Threshold tendon } \\
\text { reflexes }\end{array}$ & High & Medium & Low & $\begin{array}{l}\text { Posture of arms during trac- } \\
\text { tion test }\end{array}$ & + & ++ & +++ \\
\hline Palmar grasp & + & ++ & +++ & $\begin{array}{l}\text { Posture of head during trac- } \\
\text { tion test }\end{array}$ & + & ++ & +++ \\
\hline Plantar grasp & + & ++ & +++ & Head balance during sitting & + & ++ & +++ \\
\hline $\begin{array}{l}\text { Moro reaction, } \\
\text { intensity }\end{array}$ & + & ++ & +++ & $\begin{array}{l}\text { Spontaneous head movements } \\
\text { in prone position }\end{array}$ & + & ++ & +++ \\
\hline Ankle jerk & + & ++ & +++ & Posture in prone position & + & ++ & +++ \\
\hline Galant response & + & ++ & +++ & & & & \\
\hline
\end{tabular}

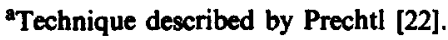


cluster score of $\leq 9$ was considered to reflect a low muscle tone, and a reflex cluster score of $\leq 10$ a low responsiveness. The cut-off points were arbitrarily chosen close to the median score.

Finally, the neurological findings were also interpreted in terms of optimality [21]. A neurological optimality score was calculated, consisting of 60 items for each of which an optimal range was defined [20]. After giving a point for each item meeting these criteria, the neurological optimality score (NOS) was calculated by the summation of optimal items. It must be emphasized that optimal is not identical with normal, and non-optimal does not always mean abnormal [21]. The neurological examinations were carried out by M.H. in Groningen and by C.K.E. in Rotterdam. Both observers were unaware of the results of the chemical analyses of the plasma and milk samples but were not blind to the feeding status.

\subsection{Samples}

Maternal blood was collected in the last month of gestation and cord blood immediately after birth. Blood was collected in a vacuum system EDTA-tube and centrifuged for $10 \mathrm{~min}$ at $3000 \mathrm{rev} . / \mathrm{min}$; plasma was stored at $-20^{\circ} \mathrm{Celsius}$ until analysis. Human milk was collected as a 24-h sample in the second and sixth week, and if possible 3 months after delivery. This was achieved by emptying both breasts with an electric pump (Babyluxus 2, KAWECO, Stuttgart, Germany). Volumes were recorded and $10 \%$ aliquots were pooled and stored at $-20^{\circ} \mathrm{Celsius}$ until analysis. The remaining milk was administered to the infants by bottle.

\subsection{Analytical methods}

Plasma samples were analyzed for the four non-planar PCB congeners 118, 138, 153 , and 180 only. Plasma has a relatively low fat content compared to human milk and too large a volume of blood would be needed to measure all the PCDD, PCDF, and PCB congeners, such as were analyzed in human milk. Plasma was denaturated with methanol and congeners were isolated from the plasma with hexane-ethyl ether. The extract was dried over sodium sulphate, concentrated and purified by chromatography over basic alumina deactivated with $10 \%$ water. A part of the cleaned extract was analyzed by gas-liquid chromatography using electron capture detection. The analysis was carried out on two capillary columns of different polarity. The limits of determination for PCB 118, 138, 153, and 180 were $0.01 \mu \mathrm{g} / 1$. The recovery of chlorinated biphenyl congeners added to the plasma before extraction, and determined as described above, was $>95 \%$. Control samples were analyzed to estimate the reliability.

The milk samples were analyzed for the seventeen 2,3,7,8-substituted PCDDs and PCDFs, which are usually found in biotic samples, three planar PCBs, and 23 nonplanar PCB congeners. Human milk was fortified with $16{ }^{13} \mathrm{C}$-labelled PCDDs and PCDFs, and three ${ }^{13} \mathrm{C}$-labelled PCB standards. The procedure for quantitative fat extraction has been described in detail, as well as the gas chromatography-highresolution mass spectrometry determination [28,29]. The non-planar PCB congeners were measured by gas chromatography using electron capture detection [30]. 


\subsection{Data processing}

In the present study, the possible toxicity of the individual congeners for the neonatal nervous system was studied in plasma and breast milk. In addition, the toxicity of a mixture of PCBs and dioxins in human milk was investigated. Finally, a comparison was made between breast-fed and formula-fed infants, in order to analyze a possible postnatal effect.

In order to express the toxic potency of the mixture of dioxins [31] and dioxin-like PCBs [32], we used the toxic equivalence factor approach. For each congener, these factors express in breast milk the relative toxicity towards $2,3,7,8-\mathrm{TCDD}$, the most toxic congener of which the toxic equivalence factor (TEF) is one. In order to calculate the toxic equivalent (TEQ) of each congener, concentrations of all 2,3,7,8compounds and planar PCBs (PCB 77, 126, and 169) were multiplied by their TEF value. By adding up the individual TEQs, a dioxin TEQ and planar PCB TEQ score could be acquired. Mono-ortho PCB TEQ (PCB 105, 118, and 156) and di-ortho PCB TEQ (PCB 170 and 180) were calculated by multiplying the concentrations and their proposed TEF value [32]. The total PCB/dioxin TEQ is a summation of the dioxin TEQ, the planar-, mono-ortho-, and di-ortho PCB TEQs.

To evaluate a postnatal effect, the sum (IPCB) of the four PCB congeners (PCB $118,138,153$, and 180 ) was calculated as a measure of $P C B$ exposure separately for cord plasma and breast milk. The $\Sigma \mathbf{S C B}_{\text {cord }}$ in cord plasma was used as a continuous independent variable, whereas the $\Sigma \mathrm{ICB}_{\text {milk }}$ in formula milk and breast milk were used as a categorial independent variable (no, low, and high postnatal exposure) in the statistical analysis.

\subsection{Statistical analysis}

The 5th, 50th, and 95th percentiles were used to describe the distribution of the concentrations. For a univariate comparison of the results in Groningen and Rotterdam, we used the chi-square test and the Wilcoxon rank sum test performed at the $5 \%$ level. Both univariate analysis and logistic regression analysis [33] were used to examine the relation between the NOS, the postural tone cluster, and the reflex cluster on one hand and levels of PCB and dioxin exposure, and obstetrical variables on the other hand. We dichotomized the NOS at the median of the pooled population. The postural tone cluster and the reflex cluster scores were dichotomized as $(\leq 9,>9)$ and $(\leq 10,>10)$, respectively. The PCB and dioxin values were logarithmically transformed. After adjusting for obstetrical variables, the effect of each chemical compound and total TEQ scores was examined. The results are reported as odds ratios (ORs) associated with doubling of concentrations, together with $95 \%$ confidence intervals $(\mathrm{CI})$, without any correction for multiple hypothesis testing.

\section{Results}

\subsection{Description of the study group}

Initially, 489 mother-infant pairs fulfilled the criteria. Seventy-one mother-infant pairs were lost mainly because of difficulties in sustaining breast-feeding, leaving a 
study population of 418 pairs. This number consisted of 104 breast-fed and 107 formula-fed infants in Groningen, and 105 breast-fed and 102 formula-fed infants in Rotterdam. Obstetrical characteristics for the total groups are presented in Table 2. No differences were found between the study centers Groningen and Rotterdam for maternal age, weight, percentage of smoking during pregnancy for both women and their partners, gender, 1-min APGAR scores and obstetrical optimality scores. In Groningen, the education of both mothers and their partners was higher compared with Rotterdam, as was the maternal alcohol consumption during pregnancy. Duration of gestation based on reported last menstrual period was also significantly different $(\mathbf{4 0 . 6} \pm 1.1$ for Groningen vs. $\mathbf{4 0 . 1} \pm 1.2$ weeks for Rotterdam), but the difference was considered to be too small to carry biological significance. In Groningen, mean birth weight was slightly higher $(3.56 \pm 0.44$ vs. $3.47 \pm 0.44 \mathrm{~kg})$.

Table 2

Baseline characteristics of the study group

\begin{tabular}{|c|c|}
\hline Variables & Outcome $(n=418)$ \\
\hline \multicolumn{2}{|l|}{ Education* } \\
\hline Lower secondary school/higher secondary school/university training (\%) & $20 / 37 / 43$ \\
\hline \multicolumn{2}{|l|}{ Parity of child } \\
\hline First born/second or third born $(\%)$ & $48 / 52$ \\
\hline \multicolumn{2}{|l|}{ Smoking during pregnancy } \\
\hline Yes/no (\%) & $26 / 74$ \\
\hline \multicolumn{2}{|l|}{ Alcohol consumption during pregnancy* } \\
\hline No/sporadic/regular (at least $1 /$ week) $(\%)$ & $72 / 25 / 3$ \\
\hline \multicolumn{2}{|l|}{ Sex of child } \\
\hline Maleffemale $(\%)$ & $54 / 46$ \\
\hline \multicolumn{2}{|l|}{ Neonatal jaundice } \\
\hline No/mild/severe (\%) & $43 / 57 / 0$ \\
\hline \multicolumn{2}{|l|}{ Maternal age (years) ${ }^{\mathrm{a}}$} \\
\hline Mean (S.D.) & $29(4)$ \\
\hline \multicolumn{2}{|l|}{ Maternal weight $(\mathrm{kg})$} \\
\hline Mean (S.D.) & $65(10)$ \\
\hline \multicolumn{2}{|l|}{ Maternal height* $(\mathrm{cm})$} \\
\hline Mean (S.D.) & $170(6)$ \\
\hline \multicolumn{2}{|l|}{ Quetelet index } \\
\hline Mean (S.D.) & 22 (3) \\
\hline \multicolumn{2}{|l|}{ Birth weight* $(\mathrm{kg})$} \\
\hline Mean (S.D.) & $3.52(0.44)$ \\
\hline \multicolumn{2}{|l|}{ Gestational age* (weeks) } \\
\hline Mean (S.D.) & $40.3(1.2)$ \\
\hline \multicolumn{2}{|l|}{ Age of examination * (days) } \\
\hline Mean (S.D.) & $14(4)$ \\
\hline \multicolumn{2}{|l|}{ Apgar $1 \mathrm{~min}$} \\
\hline Median (range) & $9(3-10)$ \\
\hline \multicolumn{2}{|l|}{ Obstetrical Optimality Score } \\
\hline Median (range) & $64(50-70)$ \\
\hline
\end{tabular}

*Significant difference between Groningen and Rotterdam $(P<0.05)$.

${ }^{2}$ In the logistic regression analysis categorised as $18-26,27-29,30-32,33-39$ years. 


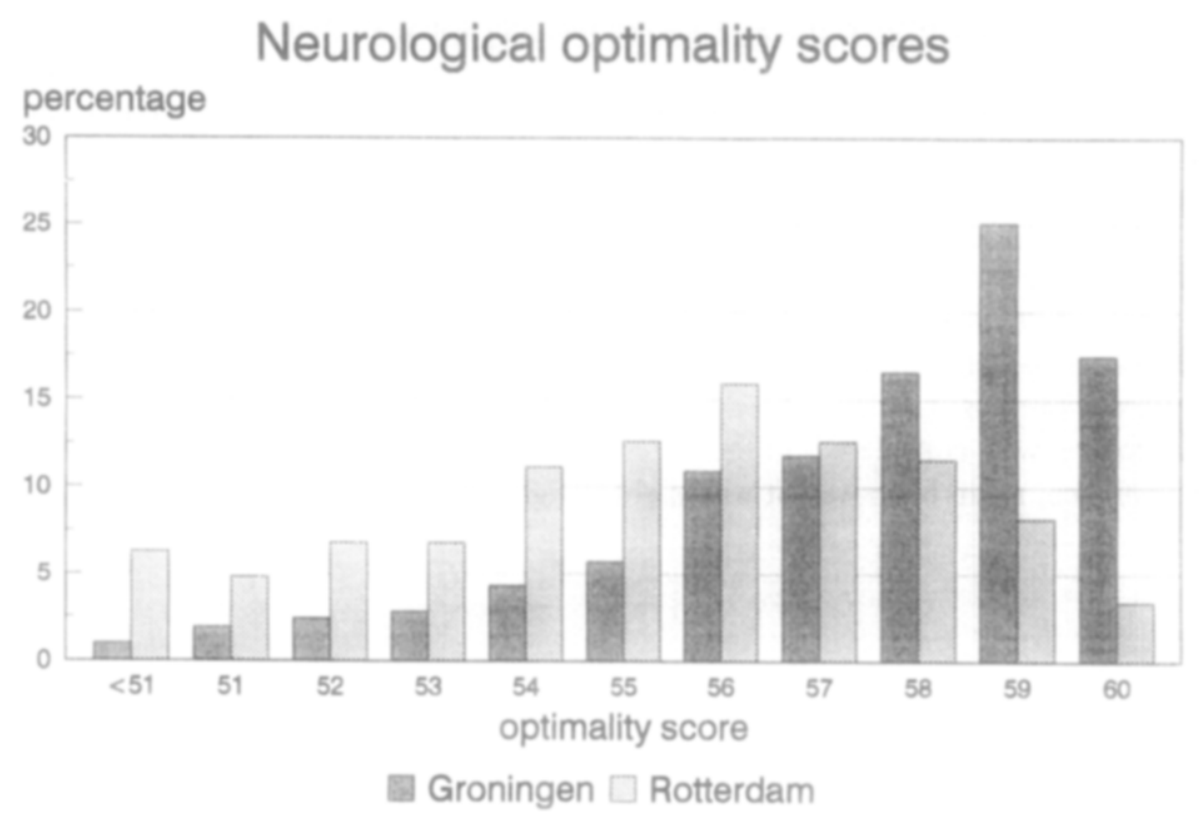

Fig. 1. Distribution of the neonatal neurological optimality scores for Groningen and Rotterdam.

Table 3

Results of logistic regression analysis ${ }^{a}$ : neonatal neurological optimality ${ }^{b}$ in relation to maternal and cord plasma levels of PCB 118, 138, 153, and 180 (International Union of Pure and Applied Chemistry (IUPAC) nomenclature)

\begin{tabular}{|c|c|c|c|c|c|c|c|}
\hline \multirow[t]{2}{*}{ Congener } & \multirow[t]{2}{*}{ IUPAC } & \multirow[t]{2}{*}{$n$} & \multicolumn{3}{|c|}{ Concentration percentiles } & \multicolumn{2}{|c|}{ Relation with NOS } \\
\hline & & & P5 & P50 & P95 & $\begin{array}{l}\text { Odds } \\
\text { ratio }\end{array}$ & $\begin{array}{l}95 \% \text { confidence } \\
\text { interval OR }\end{array}$ \\
\hline \multicolumn{8}{|l|}{ Maternal plasma $(\mu \mathrm{g} / \mathrm{I})$} \\
\hline $2,4,5,3^{\prime}, 4^{\prime} \cdot \mathrm{PECB}$ & 118 & 415 & 0.07 & 0.15 & 0.31 & 1.05 & $0.76-1.45$ \\
\hline $2,3,4,2^{\prime}, 4^{\prime}, 5^{\prime}-\mathrm{HXCB}$ & 138 & 415 & 0.27 & 0.56 & 1.10 & 1.19 & $0.81-1.73$ \\
\hline $2,4,5,2^{\prime}, 4^{\prime}, 5^{\prime} \cdot \mathrm{HXCB}$ & 153 & 415 & 0.40 & 0.84 & 1.70 & 1.08 & $0.73-1.61$ \\
\hline $2,3,4,5,2^{\prime}, 4^{\prime}, 5^{\prime}-\mathrm{HPCB}^{\prime}$ & 180 & 415 & 0.25 & 0.50 & 0.94 & 1.07 & $0.72-1.58$ \\
\hline$\Sigma P B_{\text {maternal }}{ }^{c}$ & & 415 & 1.00 & 2.04 & 3.81 & 1.11 & $0.74-1.65$ \\
\hline \multicolumn{8}{|l|}{ Cord plasmn ( $\mu g / 1)$} \\
\hline $2,4,5,3^{\prime}, 4^{\prime}-\mathrm{PECB}$ & 118 & 373 & 0.02 & 0.04 & 0.09 & 1.06 & $0.76-1.47$ \\
\hline $2,3,4,2^{\prime}, 4^{\prime}, 5^{\prime}-\mathrm{HXCB}$ & 138 & 382 & 0.04 & 0.11 & 0.25 & 1.09 & $0.81-1.48$ \\
\hline $2,4,5,2^{\prime}, 4^{\prime}, 5^{\prime}-\mathrm{HXCB}$ & 153 & 382 & 0.06 & 0.15 & 0.34 & 1.05 & $0.77-1.45$ \\
\hline $2,3,4,5,2^{\prime}, 4^{\prime}, 5^{\prime}-\mathrm{HPCB}$ & 180 & 382 & 0.04 & 0.08 & 0.19 & 1.02 & $0.75-1.39$ \\
\hline$\Sigma \mathrm{PCB}_{\text {cord }}^{\mathrm{c}}$ & & 373 & 0.18 & 0.38 & 0.86 & 0.96 & $0.68-1.36$ \\
\hline
\end{tabular}

${ }^{a}$ After adjusting for obstetrical factors.

'Dichotomized NOS: optimal, 0; non-optimal, 1.

${ }^{\mathrm{C}} \mathrm{EPCB}=\mathrm{PCB} 118+138+153+180$. 


\subsection{Neurological findings}

Sixty-three percent of the newborns were examined in the second postnatal week, $31 \%$ in the third week, and $6 \%$ in the fourth week of life. From the 418 children, 394 were neurologically classified as normal, 20 newborns as suspect, and 4 newborns as abnormal. There were no differences in the clinical diagnoses between the two study centers; these data will be reported elsewhere. The percentages of infants with a postural tone cluster score of $\leq 9$ and a reflex cluster score of $\leq 10$ were $43 \%$ and $22 \%$, respectively. The distribution of the NOS in Groningen and Rotterdam is shown in Fig. 1. In Rotterdam, the optimality score was shifted to the left compared to Groningen. There appeared to be a systematic difference between the two observers in the assessment of nine items: stability of states; posture in supine position; abdominal skin reflex; active power; knee jerk; posture of head during traction test; Moro reaction, amplitude of abduction and extension; Bauer response; and Galant response. In Groningen, these items (except for active power) were more often considered optimal. Therefore, in the logistic regression model, we adjusted for the study center. The median NOS of the pooled population used as the cut-off point was 57; the score 57 or higher was considered optimal. The NOS of the 24 neonates who were clinically diagnosed as suspect or as abnormal were all but one below the median.

\subsection{PCBs, PCDDs, and PCDFs}

The 5th, 50th, and 95th percentiles of PCB 118, 138, 153, and 180 congener concentrations in cord and plasma are shown in Table 3. The percentiles of the PCB, PCDD, and PCDF contents in breast milk are presented in Tables 4 and 5 [34]. In Rotterdam, some congener levels were higher than in Groningen [35]. Three maternal plasma samples were missing. In 382 cord plasma samples, concentrations of PCB 138, 153, and 180 congeners were analyzed. For the analysis of PCB 118 in cord plasma, nine samples were missing. In human milk, representative dioxin, planar and non-planar PCB congeners were available in 176, 194 and 195 milk samples, respectively. The Spearman correlation between $\mathbf{\Sigma P C B}_{\text {cord }}$ levels in cord plasma and $\Sigma \mathrm{PCB}_{\text {milk }}$ in human milk was $0.68(P<0.001)$. In the formula milk, we found PCB and dioxin levels below limits of determination.

\subsection{NOS and clusters versus PCBs in maternal and cord plasma}

The results of logistic regression analysis with the dichotomized NOS as the dependent variable in relation to PCB $118,138,153$, and 180 in maternal as well as in cord plasma are shown in Table 3. After adjusting for the age of the mother, the study center, alcohol consumption, and an interaction between age and alcohol consumption, no significant relation was found between the NOS and PCB congeners in maternal or cord plasma. Plasma PCB levels affected neither the cluster scores for reflexes and responses nor for postural tone.

\subsection{NOS and clusters versus PCBs, PCDDs, and PCDFs in human milk}

In breast milk, logistic regression analysis with covariate adjustment showed significant effects on the NOS of some PCB and dioxin congeners: five PCDD. two 


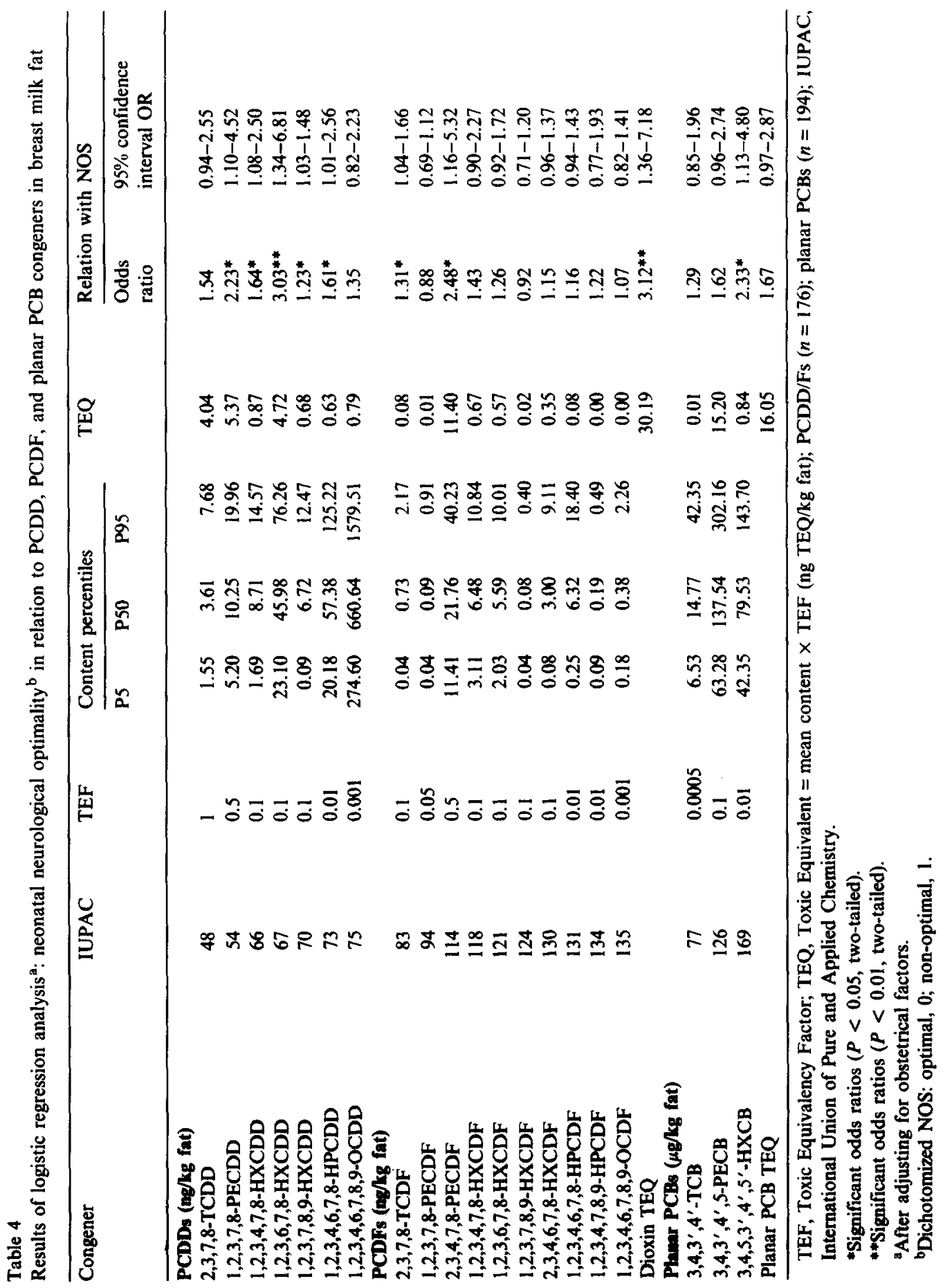


PCDF, one planar PCB, two mono-ortho PCB, one di-ortho PCB, and seven nonplanar PCB congeners as well as the dioxin, mono-ortho PCB, di-ortho PCB, and total PCB/dioxin TEQ values (Tables 4,5). After adjusting for the study center, logistic regression analysis with the postural tone cluster score as the dependent variable demonstrated a significantly higher percentage of hypotonia with an increase in planar PCB TEQ (OR: 1.64, 95\% CI: 1.03-2.63). No effect on the reflex cluster score was found.

After adjusting for the study center and for the $\Sigma \mathbf{P C B}_{\text {cord }}$ in cord plasma, a reduced neonatal neurological optimality and a higher prevalence of hypotonia was found in the highest exposed group at a turning point at $540 \mathrm{ng} \Sigma \mathrm{PCB}_{\text {milk }} / \mathrm{g}$ milk fat. The percentage of breast-fed infants with a $\Sigma P C B_{\text {milk }}$ content of $\geq 540 \mathrm{ng} / \mathrm{g}$ milk fat was $23 \%$. In this group, the odds ratio for the NOS was $3.4(95 \% \mathrm{CI}$ : 1.6-7.1). Such an odds ratio corresponds, for example, to an increase in the prevalence of non-optimality from 50 to $75 \%$.

\section{Discussion}

The neurological optimality of 418 Dutch newborns was evaluated with the use of a comprehensive age-adequate neurological examination [22]. PCB levels in cord and maternal plasma were assumed to be a direct index of prenatal PCB exposure, whereas PCB and dioxin levels in breast milk in the second week after delivery were expected to reflect the extent of intrauterine and neonatal exposure during the first 2 weeks after birth [5]. PCB levels in cord and maternal plasma were not related to (mild) nervous system dysfunction. Higher levels of PCB, PCDD, and PCDF congeners in breast milk were found to be significantly related to reduced neonatal neurological optimality. Higher levels of planar PCBs in breast milk were associated with a higher incidence of hypotonia.

The study cohort consisted of infants born in Groningen, a semi-urban area, and infants born in Rotterdam, a highly industrialized region. The two populations were obstetrically comparable. On the basis of the inclusion criteria, the study group can be considered as a 'low-risk' population for neurological abnormality. In order to specify subtle dysfunctions of the neonate's nervous system, Prechtl's optimality concept was used [21]. The neurological optimality score in Groningen was higher than in Rotterdam. It is likely that this difference is mainly due to a systematic difference in the assessment of some items by the two observers. The logistic regression analysis attempts to correct for this systematic dissimilarity (Tables 3-5).

Our neurological findings are consistent with the two studies in the United States. Rogan et al. [17] followed 912 infants with whom prenatal exposure to background levels of PCBs was estimated on the basis of the concentration of PCBs in fat of their mother's breast milk. On the BNBAS, the highly exposed newborns were found to be more hypotonic and to have a higher incidence of abnormally weak reflexes. We could confirm these results as far as the hypotonia is concerned. In a study in Michigan of 312 neonates, Jacobson et al. [16] reported a significant effect of maternal consumption of PCB-contaminated fish on neonatal neurobehavioural performance, described as motor immaturity, more limited lability of states, and weak 


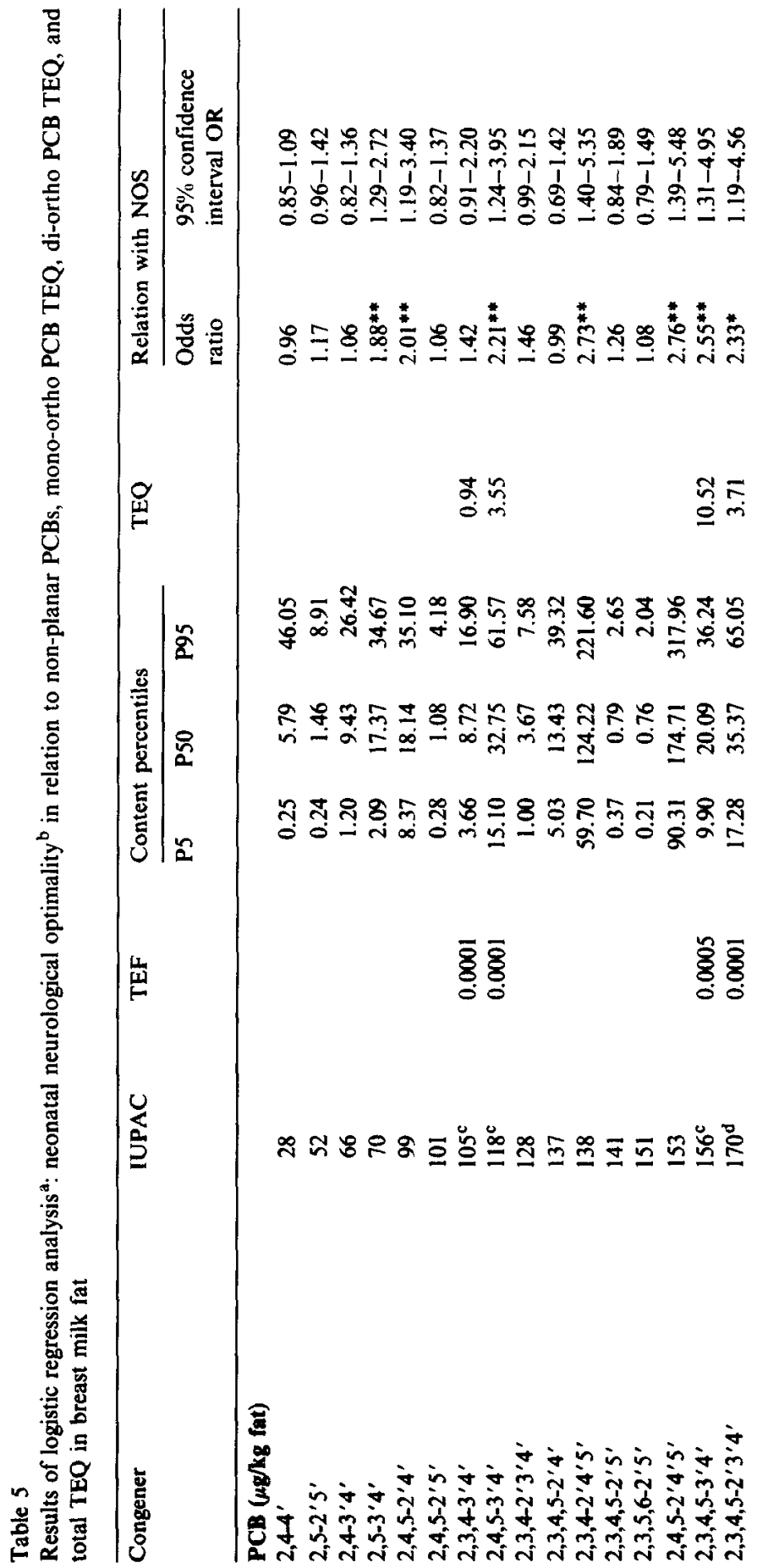




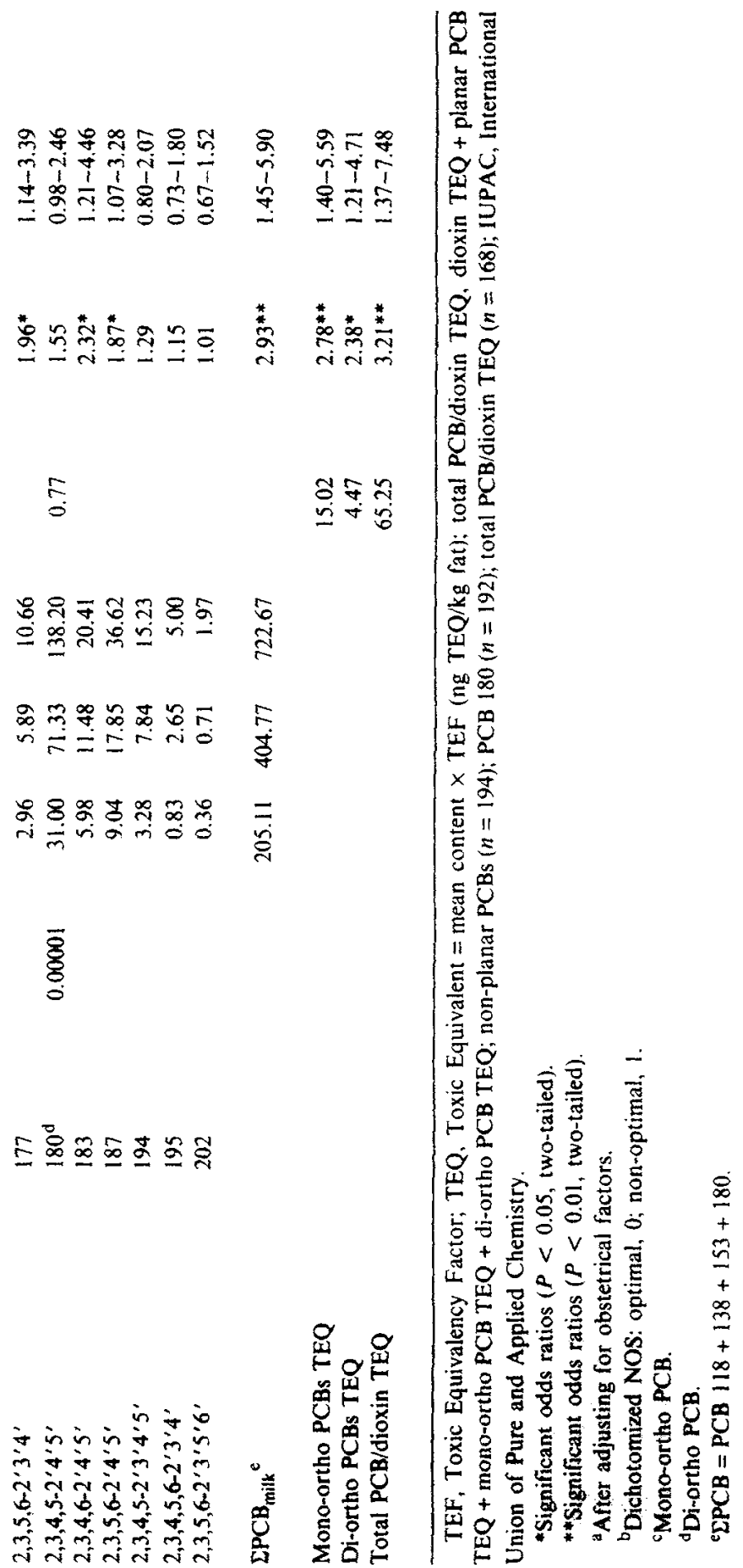


reflexes. It is essential to note that in that study the patients were examined on the third day after delivery. Beintema [36] demonstrated that neurological examinations on the first $\mathbf{2}$ or $\mathbf{3}$ days after birth have less predictive value, than those on later days of the neonatal period, because of the infant's adaptation to extrauterine life. In Michigan, the behavioural deficits were correlated to maternal fish consumption, but could not be predicted by PCB levels in cord blood. In the present study, only in breast milk fat did PCB levels show significant negative effects on the NOS. Although our congener-specific analysis differs from the total PCB determinations performed in the USA studies, the estimated levels of exposure on the basis of maternal milk samples are reasonably comparable.

Jacobson et al. [37] suggested that the central nervous system would be more vulnerable prenatally to teratogenic agents than postnatally. In the present study, the correlation between $\Sigma \mathbf{P C B}_{\text {cord }}$ levels in cord plasma and $\Sigma \mathrm{SCB}_{\text {milk }}$ in human milk was fairly strong. We calculated that the total amount of PCB 118, 138, 153, and 180 ( $\mathrm{PCB}$ ) reaching the fetus via the transplacental route is similar to that ingested

\section{Hypothetical model}

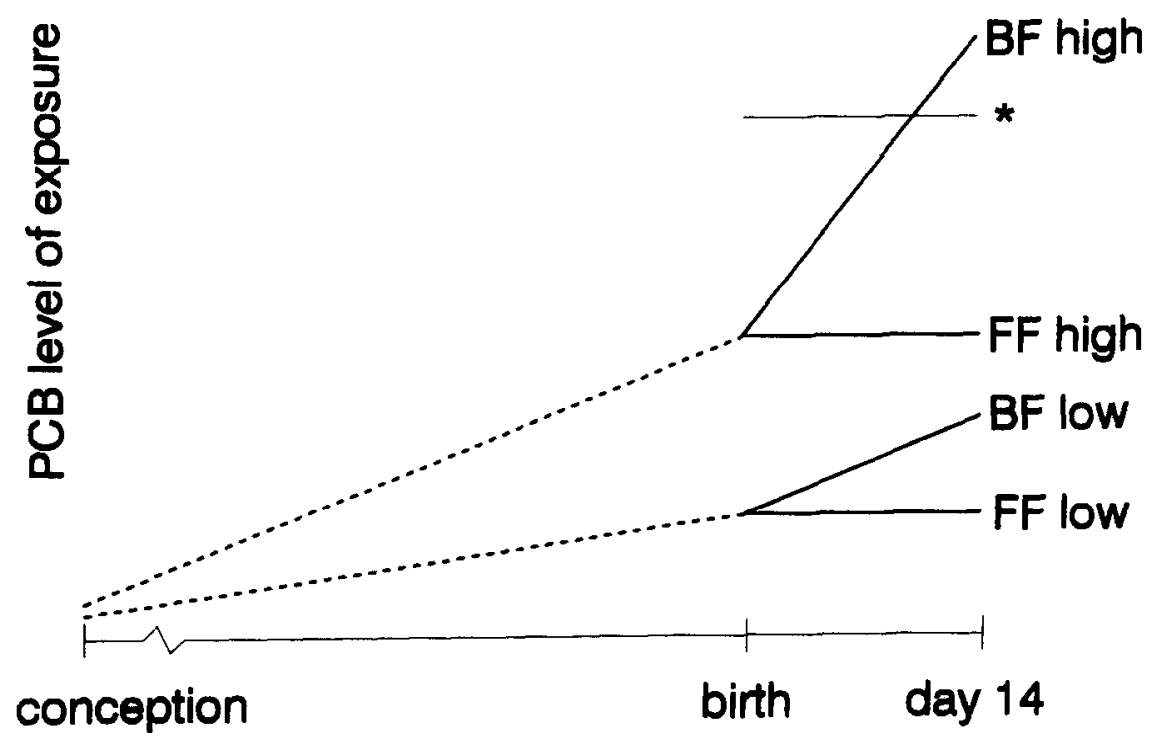

Fig. 2. Hypothetical model of the relation between the summation of intrauterine PCB exposure (PCB levels in cord plasma), and early postnatal PCB exposure (from breast milk or formula milk) versus neurological signs in the newborn period. BF high, high PCB content in plasma and breast milk exceeds critical level: neurological signs; BF low, low PCB content in plasma and breast milk does not exceed critical level: no neurological signs; FF high, high PCB content in plasma of the formula-feeding group does not exceed critical level: no neurological signs; FF low, low PCB content in plasma of the formula-feeding group does not exceed critical level: no neurological signs; "critical theoretical PCB level for neurological signs. 
by the infant during 2 weeks of breast-feeding. We found a significant negative effect of a high postnatal exposure (EPCB ${ }_{\text {milk }}>540 \mathrm{ng} / \mathrm{g}$ fat) even when prenatal exposure was adjusted for. This finding suggests an adverse effect from breast-feeding during the first 2 weeks in the presence of a high PCB level in breast milk.

Our study does not confirm prenatal effects of PCBs which have been found in other studies. It is feasible, however, that prenatal exposure in our groups remained sufficiently low, so that we did not find neurological effects in the neonatal period. Considering the high correlation between plasma and breast milk contents of PCBs, a high plasma PCB level is frequently concurrent with a high PCB level in breast milk. The combination of a high intrauterine and a high postnatal exposure might then result in neurological non-optimality as reflected by the decreased optimality score. Such a negative effect is not found in formula-fed infants with merely high plasma PCB levels (compare with Fig. 2, which shows a hypothetical model).

So in our study of a low-risk population, only breast-fed children who were perinatally exposed to higher dioxin, mono-ortho $\mathrm{PCB}$, di-ortho $\mathrm{PCB}$, and total $\mathrm{PCB} /$ dioxin TEQ values showed a reduced neonatal neurological optimality. Breastfed children exposed to higher planar PCB TEQ appeared to have a higher incidence of hypotonia. Since reflexes and responses were normal and the minor dysfunction mainly consisted of hypotonia, it is possible that the site of action is in the developing muscle. This is a subject for further study.

It should be stressed that severe neurological deviancies were absent. Considering the multitude of beneficial effects of human milk and the very minor character of the deviations, it would seem premature to advice against breast-feeding during the first weeks of life. The neonatal brain is, however, different from the brain of the older child, so that it will be necessary to investigate later neurological, but also cognitive and behavioural development, e.g. at school age.

\section{Acknowledgements}

We thank F.A.J. Muskiet and C.I. Lanting for their critical advice, Mrs A.A. Olinga for technical assistance, the midwives and the parents of the children for their co-operation. This work was supported by the Dutch Toxicology Research Promotion Programme and the Health Research Stimulation Programme.

\section{References}

[1] Kimbrough, R.D. (1974): The toxicity of polychlorinated polycyclic compounds and related chemicals. CRC Crit. Rev. Toxicol., 2, 445-498.

[2] Nondek, L. and Frolikova, N. (1991): Polychlorinated biphenyls in the hydrosphere of Czechoslovakia. Chemosphere, 23, 269-280.

[3] Bremmer, H.J. (1991): Sources of Dioxins in the Netherlands. RIVM report no. 730501014, February 1991 (in Dutch, summary in English).

[4] Kimbrough, R.D. and Jensen, A.A., (Eds.) (1989): Halogenated Biphenyls, Terphenyls, Naphthalenes, Dibenzodioxins and Related Products. Elsevier, New York.

[5] Jensen, A.A. (1987): Polychlorobiphenyls (PCBs), polychlorodibenzo-p-dioxins (PCDDs) and polychlorodibenzofurans (PCDFs) in human milk, blood and adipose tissue. Sci. Total Environ., 64, 259-293. 
[6] Theelen, R.M.C., Knaap, A.G.A.C. and Sangster, B. (1991): Dioxine: een norm aanbevolen door de Wereldgezondheidsorganisatie. Ned. Tijdschr. Geneeskd., 135, 875-877.

[7] Bowman, R.E., Heironimus, M.P. and Barsotti, D.A. (1981): Locomotor hyperactivity in PCBexposed rhesus monkeys. Neurotoxicology, 2, 251-268.

[8] Chou, S.M., Miike, T., Payne, W.M. and Davis, G.J. (1979): Neuropathology of 'spinning syndrome' induced by prenatal intoxication with a PCB in mice. Ann. N.Y. Acad. Sci., 320, 373-395.

[9] Schantz, S.L. and Bowman, R.E. (1989): Learning in monkeys exposed perinatally to 2,3,7,8tetrachlorodibenzo-p-dioxin (TCDD). Neurotoxicol. Teratol., 11, 13-19.

[10] Shain, W., Bush, B. and Seegal, R.F. (1991): Neurotoxicity of polychlorinated biphenyls: structureactivity relationship of individual congeners. Toxicol. Appl. Pharmacol., 111, 33-42.

[11] Tilson, H.A., Davis, G.J., McLachlan, J.A. and Lucier, G.W. (1979): The effects of polychlorinated biphenyls given perinatally on the neurobehavioral development of mice. Environ. Res., 18, 466-474.

[12] Seegal, R.F. (1992): Perinatal exposure to Aroclor 1016 elevates brain dopamine concentrations in the rat. Toxicologist, $12,320$.

[13] Morse, D.C., Groen, D., Veerman, M. et al. (1993): Interference of polychlorinated biphenyls in hepatic and brain thyroid hormone metabolism in fetal and neonatal rats. Toxicol. Appl. Pharmacol., 122, 27-33.

[14] Harada, M. (1976): Intrauterine poisoning: clinical and epidemiological studies and significance of the problem. Bull. Inst. Constit. Med. (Kumamoto Univ.), 25 (suppl.), 1-69.

[15] Rogan, W.J., Gladen, B.C., Hung, K.-L. et al. (1988): Congenital poisoning by polychlorinated biphenyls and their contaminants in Taiwan. Science, 241, 334-336.

[16] Jacobson, J.L., Jacobson, S.W., Schwartz, P.M., Fein, G.G. and Dowler, J.K. (1984): Prenatal exposure to an environmental toxin: a test of the multiple effects model. Dev. Psychol., 20, 523-532.

[17] Rogan, W.J., Gladen, B.C., McKinney, J.D. et al. (1986): Neonatal effects of transplacental exposure to PCBs and DDE. J. Pediatr., 109, 335-341.

[18] Brazelton, T.B. (1973): Neonatal Behavioral Assessment Scale. Lippincott, Philadelphia, PA.

[19] World Health Organization - Regional Office for Europe. (1989): Levels of PCBs, PCDDs, and PCDFs in Breast Milk. Environmental Health Series no. 34, Editor: E.J. Yrjänheikki, WHO, Geneva.

[20] Touwen, B.C.L., Huisjes, H.J., Jurgens-van der Zee, A.D. et al. (1980): Obstetrical condition and neonatal neurological morbidity. An analysis with the help of the optimality concept. Early Hum. Dev., 4, 207-228.

[21] Prechtl, H.F.R. (1980): The optimality concept. Early Hum. Dev., 4, 201-205.

[22] Prechtl, H.F.R. (1977): The Neurological Examination of the Full-term Newborn Infant, 2nd edn. Clinics in Developmental Medicine, No. 63, SIMP. Heinemann, London.

[23] Sameroff, A.J. (1978): Summary and conclusions: the future of newborn assessment. In: Monographs of the Society for Research in Child Development: Organization and Stability of Newborn Behaviour Assessment Scale, Serial No. 177, vol. 43, pp. 102-103. Editor: A.J. Sameroff, Chicago University Press, Chicago.

[24] Bierman-van Eendenburg, M.E.C., Jurgens-van der Zee, A.D., Olinga, A.A., Huisjes, H.J. and Touwen, B.C.L. (1981): Predictive value of neonatal neurological examination: a follow-up study at 18 months. Dev. Med. Child Neurol., 23, 296-305.

[25] Hadders-Algra, M., Touwen, B.C.L. and Huisjes, H.J. (1986): Neurologically deviant newborns: neurological and behavioural development at the age of six years. Dev. Med. Child Neurol., 28, 569-578.

[26] Hadders-Algra, M., Huisjes, H.J, and Touwen, B.C.L. (1988): Perinatal correlates of major and minor neurological dysfunction at school age: a multivariate analysis. Dev. Med. Child Neurol., 30, $472-481$.

[27] Touwen, B.C.L., Lok-Meijer, T.Y., Huisjes, H.J. and Olinga, A.A. (1982): The recovery rate of neurologically deviant newborns. Early Hum. Dev., 7, 131-148.

[28] Van Rhijn, J.A., Traag, W.A., Kulik, W. and Tuinstra, L.G.M.Th. (1992): Automated clean-up procedure for the gas chromatographic-high-resolution mass spectrometric determination of polychlorinated dibenzo-p-dioxins and dibenzofurans in milk. J. Chromatogr., 595, 289-299. 
[29] Van Rhijn, J.A., Traag, W.A., van de Spreng, P.F. and Tuinstra, L.G.M.Th. (1993): Simultaneous determination of planar chlorobiphenyls and polychlorinated dibenzo-p-dioxins and -furans in Dutch milk using isotope dilution and gas chromatography-high-resolution mass spectrometry. $\mathrm{J}$. Chromatogr., 630, 297-306.

[30] Tuinstra, L.G.M.Th., Van Rhijn, J.A., Traag, W.A. et al. (1993): Method for the determination of dioxins, planar and other PCBs in human milk. Halogenated Compounds 11, 181-183.

[31] NATO, Committee on the Challenges of Modern Society. Pilot study on International Information Exchange on Dioxins and Related Compounds. International Toxicity Equivalence Factor (I-TEF). Method of Risk Assessment for Complex Mixtures of Dioxins and Related Compounds. Report number 176, Brussels, NATO, 1988.

[32] Ahlborg, U.G., Becking, G.C., Birnbaum, L.S. et al. (1994): Toxic equivalency factors for dioxinlike PCBs. Chemosphere, 28, 1049-1067.

[33] Hosmer, D.W. and Lemeshow, S. (1989): Applied Logistic Regression. Editors: D.W. Hosmer and S. Lemeshow, Wiley, New York.

[34] Koopman-Esseboom, C., Huisman, M., Weisglas-Kuperus, N. et al. (1994): PCB and dioxin levels in plasma and human milk of $\mathbf{4 1 8}$ Dutch women and their infants. Predictive value of PCB congener levels in maternal plasma for fetal and infant's exposure to PCBs and dioxins. Chemosphere, 28 , $1721-1732$.

[35] Koopman-Esseboom. C., Huisman, M., Weisglas-Kuperus, N. et al. (1994): Dioxin and PCB levels in blood and human milk in relation to living areas in the Netherlands. Chemosphere, submitted.

[36] Beintema, D.J. (1968): A Neurological Study of Newborn Infants. In: Clinics in Developmental Medicine, No. 28. Heinemann, London.

[37] Jacobson, J.L., Fein, G.G., Jacobson, S.W., Schwartz, P.M. and Dowler, J.K. (1984): The transfer of polychlorinated biphenyls (PCBs) and polybrominated biphenyls (PBBs) across the human placenta and into maternal milk. J. Public Health, 74, 378-379. 